\title{
CORSIKA modification for rigidity dependent primary selection based on geomagnetic cut-off rigidity for GRAPES-3 simulations
}

\author{
B. Hari Haran ${ }^{1, a, b}$, S. Ahmad ${ }^{a, c}$, A. Chandra ${ }^{a, b}$, S.R. Dugad ${ }^{a, b}$, S.K. Gupta ${ }^{a, b}$, Y. \\ Hayashi $^{\mathrm{a}, \mathrm{d}}$, A. Jain ${ }^{\mathrm{a}, \mathrm{b}}$, S. Kawakami ${ }^{\mathrm{a}, \mathrm{d}}$, P.K. Mohanty ${ }^{\mathrm{a}, \mathrm{b}}$, A. Oshima ${ }^{\mathrm{a}, \mathrm{e}}$, P. \\ Jagadeesan $^{\mathrm{a}, \mathrm{b}}$, B.S. Rao ${ }^{\mathrm{a}, \mathrm{b}}$ \\ ${ }^{a}$ GRAPES-3 Experiment, Cosmic Ray Laboratory, Ooty 643 001, India. \\ ${ }^{b}$ Tata Institute of Fundamental Research, Homi Bhabha Road, Mumbai 400 005, India. \\ ${ }^{c}$ Department of Physics, Aligarh Muslim University, Aligarh 202002, India. \\ ${ }^{d}$ Graduate School of Science, Osaka City University, Osaka 558-8585, Japan. \\ ${ }^{e}$ Chubu University, Engineering Science Laboratory, Kasugai-shi, Aichi 487-8501, Japan. \\ E-mail: 89hariharan@gmail.com
}

For the analysis of the GRAPES-3 muon data, large scale Monte Carlo simulations are required. These simulations are performed using the CORSIKA simulation package developed by the KIT group. However, the geomagnetic cut-off rigidity at a given location varies with direction, and therefore, a constant threshold for selection of primary energy results in generation of a large number of events that are subsequently rejected due to their rigidity being below the cut-off value for that direction. We have implemented an efficient mechanism in CORSIKA to select only those primary cosmic rays that lie above the cut-off rigidity in a given direction resulting in rejection of the primary cosmic rays that would have otherwise been rejected subsequently. Results based on actual simulations of GRAPES-3 muon data have shown that by using this rigidity based cut, the actual computation time was reduced by a factor of two without affecting the final results.

The 34th International Cosmic Ray Conference

30 July- 6 August, 2015

The Hague, The Netherlands

${ }^{1}$ Speaker 


\section{Introduction}

The simulations for GRAPES-3 data analysis were optimized by introducing a rigidity based cut in the CORSIKA package [1]. This modification selects only those primary particles that are required for GRAPES-3 simulation. Since this selection happens before the simulation process starts, simulation of unwanted primaries which would have been subsequently rejected, is avoided. This real-time selection is based on pre-calculated cut-off rigidities for field of view at GRAPES-3 location. No change in physics results were observed with this modified version of CORSIKA when compared with the results obtained using the original code.

\section{Cosmic Rays and Air Showers}

The cosmic ray energy spectrum extends over many order of magnitude, to energies up to $10^{20} \mathrm{eV}$. The primary spectrum follows a power law which falls with rising energy about like $\alpha$ $\mathrm{E}^{-2.7}$. Since cosmic rays are charged, they are deflected in galactic \& intergalactic magnetic fields such that their arrival directional information is completely lost. The cosmic rays above $10^{13} \mathrm{eV}$ can be detected via their interaction in the atmosphere which produces large number of secondaries scattered over large area on the ground. The energy and mass of the primary cosmic ray has to be deduced from the measured properties of air shower. The shower development does not depend only on the energy and mass of the primary cosmic ray, but also on how it interacts in the atmosphere by means of electromagnetic and hadronic interactions, the particle transport and decay and on statistical fluctuations in each process.

\section{Need of simulations}

A simulation is a model of system which can be described using an algorithm. A model is a simplified description of a particular system or process. The system may be a real physical system or a theoretical model. Usually, a simulation is based upon a mathematical model in which input parameters are fed and certain outputs are produced. Since most of the simulations are based on a mathematical model that can be programmed to run on computers. Large and complex problems can usually be dissected into smaller and simpler sub-problems. The simulation then provides a numerical convolution of these individual parts to a greater and more complex whole. If the sub-problems are known in all details, then the numerical simulation produces the correct result, including all correlations. If all details are not known then it is impractical to do a full simulation. Then realistic models are used with several simplifications, assumptions or approximations. Then more care is needed to ensure that the models do not affect the results. Therefore, the simulation of elementary processes is the method of choice for a complex problem, such as the formation of air showers in the atmosphere.

In EAS experiments the measurable quantities such as number of secondary particles at detector level, lateral energy distribution, arrival time and Cherenkov light production etc. are used to estimate the primary energy, mass and direction. The analysis and interpretation of such experimental data needs detailed Monte Carlo simulation of multi-parameterized function of energy, mass and direction. It is important to study the shower development in the atmosphere to 
understand the characteristic of the primary cosmic ray and the measurable quantities at the observation level. To draw quantitative conclusions from observed distributions it is necessary to model the air showers in detail. The detailed Monte Carlo simulation of air shower development in the atmosphere is a complicated process because it requires the consideration of many factors that are capable of affecting the air showers.

The basis of air shower simulation is the Monte Carlo event generators which deals different hadronic interactions. These generate events for particle production in the atmosphere from the primary cosmic ray.

\section{GRAPES-3 muon telescope}

A large area $\left(560 \mathrm{~m}^{2}\right)$ muon tracking detector of the GRAPES-3 experiment [2], operating at Ooty, India has been designed to study high energy cosmic ray astrophysics. The muon detector is used to differentiate primary gamma rays from charged cosmic rays and to obtain cosmic ray composition, and probe various solar phenomena.

The GRAPES-3 muon detector uses proportional counter (PRC) as basic element. Each PRC is $600 \mathrm{~cm}$ long, $10 \mathrm{~cm} \mathrm{X} 10 \mathrm{~cm}$ cross section with a wall thickness of $2.3 \mathrm{~mm}$. A muon detector module consists of 232 PRCs arranged in 4 layers, with alternate layers placed in mutually orthogonal directions which gives an sensitive area of $35 \mathrm{~m}^{2}$. Two successive layers of PRCs are separated by $15 \mathrm{~cm}$ thick concrete. The muon detector permits a two-dimensional reconstruction of muon tracks in two vertical, orthogonal planes. The vertical separation of two layers of PRCs in the same plane is $50 \mathrm{~cm}$ which allows the muon track direction to be measured to an accuracy of $6^{\circ}$ in the projected plane. To achieve an energy threshold of $1 \mathrm{GeV}$ for vertical muons, a total thickness $\sim 550 \mathrm{~g} \mathrm{~cm}^{-2}$ in the form of concrete blocks of $2.4 \mathrm{~m}$ thickness is used as absorber. The concrete blocks have been arranged in the shape of an inverted pyramid to achieve an energy threshold of $1(\sec \theta) \mathrm{GeV}$ for muons incident at a zenith angle $\theta$ (with coverage up to $45^{\circ}$ ). Four muon detector modules form a super-module.

\section{Introduction to CORSIKA}

CORSIKA is a Monte Carlo program to study the development of extensive air showers in the atmosphere from $10^{9} \mathrm{eV}$ to $10^{20} \mathrm{eV}$. It was developed for the KASCADE experiment [3] at Karlsruhe, Germany. There are many other simulation programs like CRY [4] \& AIRES [5] to simulate the propagation of cosmic rays in the atmosphere. CORSIKA is one of the most widely accepted Monte Carlo simulator used by the cosmic ray community. It performs 4-dimensional simulations and includes the interaction and decay of nuclei, hadron, muons, electrons and photons in the atmosphere. These particles are tracked through the atmosphere until they undergo interactions or decay into secondary particles. The particle information can be obtained for a given observation level. The main CORSIKA program contains $\sim 78000$ lines of FORTRAN and $\mathrm{C}$ routines which are optional. CORSIKA can be used with various hadronic event generators which are interfaced with main CORSIKA program that can be activated by the user. It uses models such as DPMJET [6], EPOS LHC [7], NEXUS [8], QGSJET 01C [9], QGSJETII-04 [10], SIBYLL 2.1 [11,12] \& VENUS 4.12 [13] for high energy hadronic interactions and GHEISHA [14], FLUKA [15] \& UrQMD [16] for low energy hadronic interactions. 


\section{GRAPES-3 simulations}

GRAPES-3 muon telescope records $4 \times 10^{9}$ muons everyday. These muons are recorded in 225 angular bins. For a comparative study of these muons, billions of cosmic rays showers need to be generated using Monte Carlo programs to obtain secondary muon information. These simulations require a long time to complete with presently available computing power. Therefore, an efficient scheme to minimize simulation time is an essential requirement of every experiment. The GRAPES-3 experiment is sensitive to cosmic rays of energies from $10 \mathrm{GeV}$ to $10 \mathrm{TeV}$. But the energy threshold is direction dependent and changes due to geomagnetic effects that are explained in the subsequent section.

\section{Geomagnetic cut-off rigidity}

The charged particles originating from outside the Earth's magnetosphere are deflected by the Lorentz force. Thus, the ability of a particle to penetrate the geomagnetic field depends upon the magnetic rigidity $(\mathrm{R})$ which is defined as particle momentum $(\mathrm{pc})$ per unit charge $(\mathrm{q})$.

$$
R=\frac{p c}{q}(G V)
$$

Rigidity $\mathrm{R}$ is a useful parameter to describe the motion of charged particles in the geomagnetic field. For any point in the magnetosphere, a minimum rigidity called cut-off rigidity defines the threshold to reach that point. Particles with rigidity lower than the cut-off rigidity will be deflected back into space, whereas particles above the cut-off rigidity will reach that point.

The cut-off rigidity map for GRAPES-3 location is given in Fig-1. The angular variation of cut-off rigidity can be seen clearly.

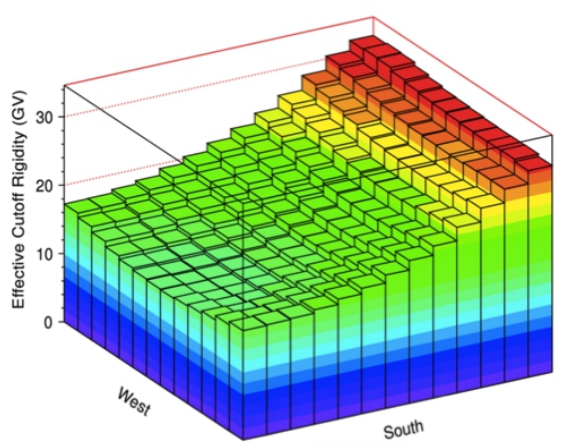

Fig-1 cut-off rigidity 3D map

\section{CORSIKA modifications for primary selection}

We have used officially released CORSIKA v7.4000 for our modification. CORSIKA program flow and the modifications are shown in Fig-2. A look-up table was created for cut-off rigidity values for GRAPES-3 location using IGRF-11 model [17] with zenith ranges from 0$60^{\circ}$ and azimuth ranges from $0-360^{\circ}$ in $1^{\circ}$ bin width. After the initialization of CORSIKA variables, we have introduced few more variables which do not affect the final outcome. A lookup table containing geomagnetic cut-off values is loaded into the CORSIKA memory. After the primary parameters (energy, direction) are calculated in CORSIKA with modified code which 


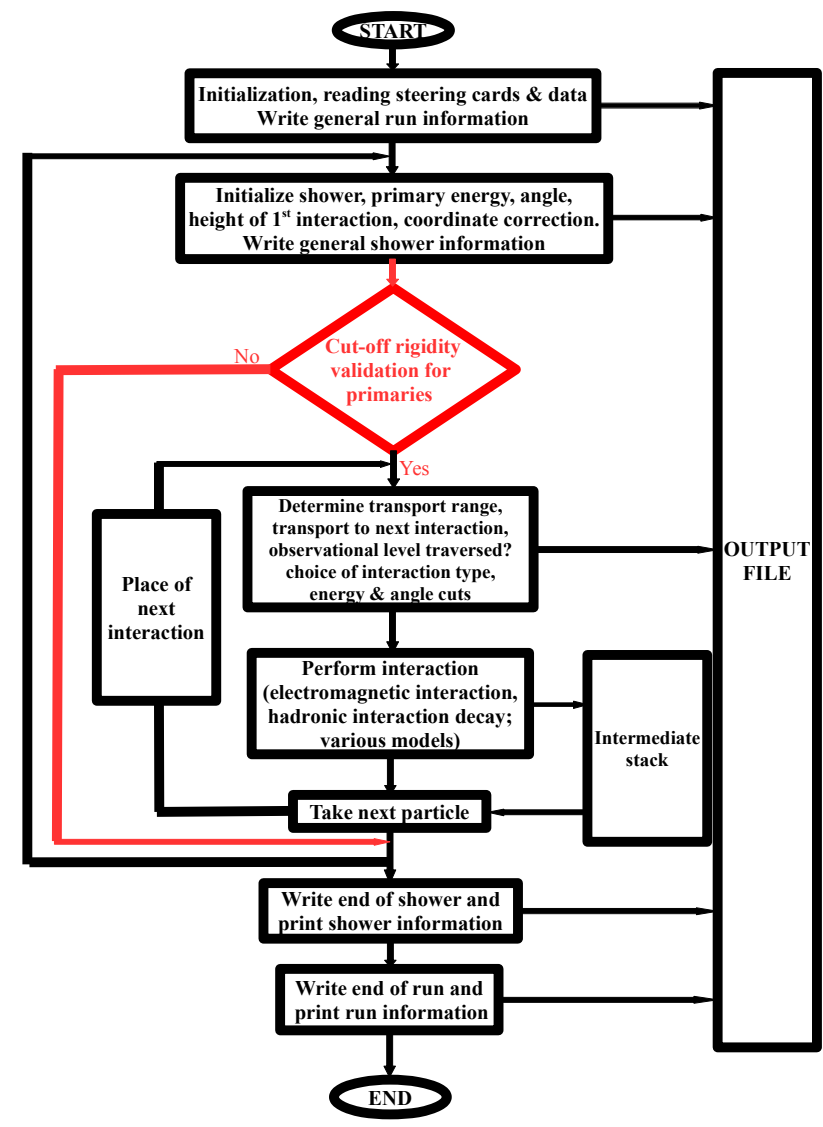

Fig-2

CORSIKA Program flow Modified program flow

validates the generated primary passes the cut-off rigidity in that direction. If the validation is met then only the primary can undergo for normal CORSIKA process or else it will be rejected there itself.

\section{Results}

\begin{tabular}{|c|c|c|}
\hline PARAMETERS & $\begin{array}{c}\text { WITHOUT } \\
\text { MODIFICATION }\end{array}$ & $\begin{array}{c}\text { WITH } \\
\text { MODIFICATION }\end{array}$ \\
\hline Total run time (Sec) & 2424 & 1238 \\
\hline DATnnnnnn (MByte) & 239 & 100 \\
\hline DATnnnnnn.long (MByte) & 950 & 357 \\
\hline Events generated & 100000 & 36942 \\
\hline
\end{tabular}

Table-1 Results of modification,

Parameters compared with original code

In earlier procedure, the primary particles were generated and the shower selection was done offline which resulted in significant computing resources being wasted. For this purpose, SIBYLL and Gheisha models were used to generate $10^{5}$ proton showers with zenith angle range $0-60^{\circ}$ and azimuthal range $0-360^{\circ}$. The results of these simulations are listed in Table- 1 which shows that only $37 \%$ of the simulated showers are useful. Remaining showers that did not pass 
the cut-off rigidity condition had to be rejected. By using the rigidity dependent cut the computing time was reduced by a factor $\sim 2$ and the size of the output file was also reduced by a factor $\sim 3$. The primary energy spectrum from the simulated showers is shown in Fig-3.

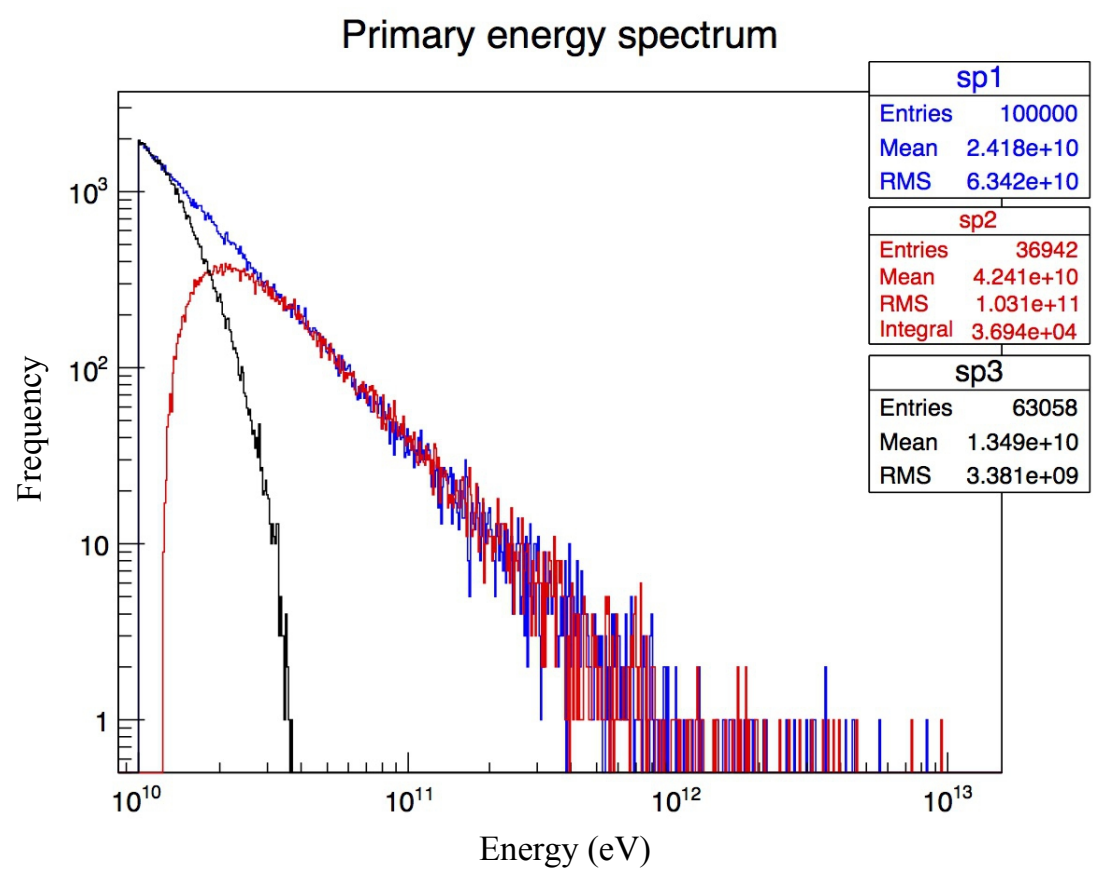

Fig-3 Primary energy spectrum

Total showers

Simulated showers

Rejected showers

\section{References}

[1] Heck, D. et al.. CORSIKA: A Monte Carlo Code to Simulate Extensive Air Showers, FZKA Report 6019.

[2] Y. Hayashi et al. Nucl. Instrum. and Methods A 545 (2005) 643-657.

[3] The Cosmic-Ray Experiment KASCADE, Nucl. Instru. And Meth A 513 (2003) 490-510.

[4] Monte Carlo Simulation of Proton-induced Cosmic-ray Cascades in the Atmosphere, UCRL-TM229452.

[5] AIRES: A system for air shower simulations, arXiv:astro-ph/9911331v1.

[6] J. Ranft, Phys. Rev. D51 (1995) 64; preprint arXiv: hep-ph/9911213 and hep-ph/9911232 (1999).

[7] T. Pierog et al., arXiv:1306.0121[hep-ph] (2013).

[8] H.J. Drescher et al., Phys. Rep. 350 (2001) 93.

[9] N.N. Kalmykov and S.S. Ostapchenko, Yad. Fiz. 56 (1993) 105; Phys. At. Nucl. 56 N3 (1993) 346; N.N. Kalmykov, S.S. Ostapchenko, and A.I. Pavlov, Izv. RAN Ser. Fiz. 58 (1994) N12 p.21; N.N.

Kalmykov, S.S. Ostapchenko, and A.I. Pavlov, Bull. Russ. Acad. Science (Physics) 58 (1994) 1966; 
N.N. Kalmykov, S.S. Ostapchenko, and A.I. Pavlov, Nucl. Phys. B (Proc. Suppl.) 52B (1997) 17; S.S. Ostapchenko, private communications (2001).

[10] S.S. Ostapchenko, Phys. Rev. D83 (2011) 014018.

[11] R.S. Fletcher, T.K. Gaisser, P. Lipari, and T. Stanev, Phys. Rev. D50 (1994) 5710; J. Engel, T.K. Gaisser, P. Lipari, and T. Stanev, Phys. Rev. D46 (1992) 5013.

[12] R. Engel, T.K. Gaisser, P. Lipari, and T. Stanev, Proc. 26th Int. Cosmic Ray Conf., Salt Lake City (USA), 1 (1999) 415; E.-J. Ahn, R. Engel, T.K. Gaisser, P. Lipari, and T. Stanev, Phys. Rev. D80 (2009) 094003; R. Engel, private communications.

[13] K. Werner, Phys. Rep. 232 (1993) 87.

[14] H. Fesefeldt, Report PITHA-85/02 (1985), RWTH Aachen, available from: http://cds.cern.ch/record/162911/files/CM-P00055931.pdf

[15] A. Ferrari, P.R. Sala, A. Fass`o, and J. Ranft, Report CERN-2005-10 (2005); INFN/TC 05/11; SLAC-R-773 (2005); A. Fass`o, A. Ferrari, S. Roesler, P.R. Sala, G. Battistoni, F. Cerutti, E. Gadioli, M.V. Garzelli, F. Ballarini, O. Ottolenghi, A. Empl, and J. Ranft, Computing in High Energy and Nuclear Physics 2003 Conference (CHEP2003), La Jolla, CA (USA), March 24-28, 2003 (paper MOMT005); eConf C0303241 (2003); arXiv: hep-ph/0306267 (2003); http://www.fluka.org/

[16] S.A. Bass et al., Prog. Part. Nucl. Phys. 41 (1998) 225; M. Bleicher et al., J. Phys. G: Nucl. Part. Phys. 25 (1999) 1859; http://www.th.physik.uni-frankfurt.de/ urqmd/

[17] International Geomagnetic Reference Field: the eleventh generation, DOI: $10.1111 /$ j.1365246X.2010.04804.X

[18] www.ph.surrey.ac.uk 